\title{
Correlation between percutaneous biopsy and final histopathology for retroperitoneal sarcoma: A single-centre study
}

Rebekah Young ${ }^{1,2}$, Hayden Snow ${ }^{1}$, Shona Hendry ${ }^{3,4}$, Catherine Mitchell ${ }^{5}$, John Slavin ${ }^{3}$, Stephen Schlicht ${ }^{6}$, Lumine $\mathrm{Na}^{7}$, Michael S. Hofman ${ }^{8,9}$, David E Gyorki ${ }^{1,9}$

${ }^{1}$ Department of Cancer Surgery, Peter MacCallum Cancer Centre, Victoria, Australia ${ }^{2}$ Melbourne Health, Victoria, Australia

${ }^{3}$ Department of Anatomical Pathology, St Vincent's Hospital, Victoria, Australia ${ }^{4}$ Department of Pathology, University of Melbourne, Victoria, Australia ${ }^{5}$ Department of Pathology, Peter MacCallum Cancer Centre, Victoria, Australia ${ }^{6}$ Department of Radiology, St Vincent's Hospital, Victoria, Australia ${ }^{7}$ Centre for Biostatistics and Clinical Trials, Peter MacCallum Cancer Centre ${ }^{8}$ Department of Molecular Imaging and Therapeutic Nuclear Medicine, Peter MacCallum Cancer Centre, Victoria, Australia

${ }^{9}$ University of Melbourne, Victoria, Australia

${ }^{10}$ Department of Surgery, University of Melbourne, Victoria, Australia

Corresponding Author:

A/Prof David Gyorki

C/O Peter MacCallum Cancer Centre, 305 Grattan St, Melbourne VIC 3000

This is the author manuscript accepted for publication and has undergone full peer review but has not been through the copyediting, typesetting, pagination and proofreading process, which may lead to differences between this version and the Version of Record. Please cite this article as doi: 10.1111/ans.15723

This article is protected by copyright. All rights reserved. 
david.gyorki@petermac.org

Word count

Abstract: 249 words

Manuscript: 2,656 words

Table and figure count

Tables: 5

Figures: 3

This article is protected by copyright. All rights reserved. 


\begin{abstract}
Background:

Retroperitoneal sarcomas are rare soft tissue tumours accounting for $10-15 \%$ of soft tissue sarcomas. Patient prognosis and treatment recommendations (including extent of surgery and neoadjuvant strategies) are determined by the preoperative histopathological subtype and grade obtained from biopsy and thus it is important to understand the accuracy of biopsy in retroperitoneal masses.
\end{abstract}

Materials and methods: This study presents a case series of primary retroperitoneal sarcomas managed at Peter MacCallum Cancer Centre (PMCC) between 2008 and 2019. Statistical analyses were performed to determine correlation between histopathology from percutaneous biopsy and surgical excision. Results: A total of 117 patients who underwent percutaneous core biopsy and surgical excision of retroperitoneal sarcoma were included. Diagnostic accuracy varied with histopathological diagnosis, but overall precise concordance between biopsy and final histopathology was seen in $61 \%(\kappa=0.57)$. Biopsy was most sensitive for identifying well differentiated liposarcoma (WDLPS) (sensitivity 85\%, $95 \% \mathrm{Cl} 0.06$ to 0.96 ) and leiomyosarcoma (sensitivity $81 \%, 95 \% \mathrm{Cl} 0.54$ to 0.96 ) and was least sensitive for identifying dedifferentiated liposarcoma (DDLPS) (sensitivity 40\%, 95\% Cl 0.25-0.56). Overall agreement between biopsy and final histopathology increased with use of PET/CT scan pre-biopsy and with use of FISH testing on biopsy, however, neither test improved recognition of dedifferentiated components within WD/DDLPS on core biopsy. 


\section{Conclusions:}

Pre-operative biopsy is important for clinical decision making in the treatment of retroperitoneal sarcoma. A significant portion of patients with a WDLPS will have a dedifferentiated component identified at the time of resection that was not identified on initial biopsy. 


\section{Introduction}

The retroperitoneum is the site of origin for approximately $10-15 \%$ of all soft tissue sarcomas with an incidence between $0.5-1$ new diagnoses per 100,000 population per year ${ }^{1}$. The primary treatment modality for the majority of patients is surgical resection, with radiotherapy or chemotherapy being selectively used in the adjuvant/neoadjuvant setting ${ }^{2}$. The differential diagnosis for retroperitoneal masses is broad and includes over 70 subtypes of retroperitoneal sarcoma (RPS) as well as a host of non-sarcomatous malignancies and a number of benign masses ${ }^{1,3}$. Patient prognosis and treatment recommendations (including extent of surgery and neoadjuvant strategies) are determined by the preoperative histopathological subtype and grade obtained from biopsy of a retroperitoneal mass and thus thorough pre-operative workup is required. Liposarcoma (LPS) is the most common type of soft tissue sarcoma overall and the most common pathological type of sarcoma occurring in the retroperitoneum. Liposarcoma can be further classified into four histologic subtypes - well differentiated (WDLPS), well differentiated/dedifferentiated (WD/DDLPS), myxoid and pleomorphic types ${ }^{4-6}$. For patients with WD/DDLPS, the presence of a de-differentiated component and its grade are the most important prognostic factors with the de-differentiated component associated with an increased risk of recurrence and mortality?

Definitive diagnosis of retroperitoneal masses can be difficult to achieve radiologically and thus percutaneous biopsy is recommended to guide diagnosis and 
treatment ${ }^{2}$. The extent of dedifferentiation in a WD/DDLPS is variable and heterogeneous and therefore, even when the diagnosis of sarcoma is made, sampling error from biopsy may result in incorrect subtype-specific diagnosis or inaccurate tumour grading.

Given the heavy reliance on pre-operative biopsy in determining management plans, it is important to establish the accuracy of percutaneous core biopsy for determining histopathology of retroperitoneal sarcomas. The aim of this study was to assess the accuracy of percutaneous biopsy for predicting final histopathological subtype for retroperitoneal sarcomas in an Australian specialist sarcoma centre and to comment on whether adjunct tests such as positron emission tomography/computed tomography (PET/CT) scan or fluorescence in situ hybridisation (FISH) testing improve diagnostic accuracy.

\section{Materials and Methods}

This study presents a case series of primary retroperitoneal sarcoma cases managed at the Peter MacCallum Cancer Centre (PMCC) between 2008 and 2019. Patients who underwent both percutaneous core biopsy and surgical resection of a retroperitoneal mass with the histopathology of one or both of these interventions suggesting a diagnosis of retroperitoneal sarcoma were included in this study. 
Patients were excluded if no pre-operative biopsy was undertaken or if an alternative biopsy technique was utilised.

Percutaneous biopsies were undertaken under radiological guidance by specialist interventional radiologists with extensive experience in sarcoma at an external site and all histopathology was reviewed by pathologists with specialisation in sarcoma. As part of standard practice, the imaging, histopathology and management of patients receiving treatment through PMCC was discussed at a sarcoma multidisciplinary meeting.

This paper classified biopsy and final histopathology results as either welldifferentiated liposarcoma (WDLPS), well differentiated/de-differentiated liposarcoma (WD/DDLPS), leiomyosarcoma (LMS), myxoid liposarcoma (myxoid LPS), undifferentiated pleomorphic sarcoma (UPS), solitary fibrous tumour (SFT), other sarcoma, non-sarcoma malignancy, benign tumour or non-diagnostic.

Data were extracted from hospital files prior to 2016 and then from a prospectively maintained registry of retroperitoneal sarcoma patients thereafter (2016-2019). Data pertaining to demographics, percutaneous biopsy results, pre-operative radiological staging, specifications of surgical resection and post-operative histopathology were collected for each patient. 
Percutaneous biopsy results were compared with final histopathology from surgical resection using a Clopper-Pearson exact test to calculate 95\% confidence intervals (Cls). Diagnostic performance was described by sensitivity, specificity, positive and negative predictive value (PPV and NPV). A weighted kappa statistics $(\kappa)$ with 95\% Cls was used to assess agreement between biopsy and final histopathology; $\kappa \leq 0$ interpreted as no agreement, $\kappa=0.01$ to 0.2 as none to slight, $\kappa=0.21$ to 0.4 as fair, $\kappa$ $=0.41$ to 0.60 as moderate, $\kappa=0.61$ to 0.80 as substantial, and $\kappa=0.81-1.00$ as almost perfect agreement ${ }^{4}$ (see table 1).

Ethical approval for this project was obtained from the Human Resources and Ethics Committee (HREC) at PMCC. Data were anonymised at the time of collection and stored in accordance with the HREC specifications.

\section{Results}

A total of 117 patients who received care at PMCC between 2008 and 2019 were included in this study (see table 2). The majority of patients underwent surgical resection at $\mathrm{PMCC}$, however 9 patients had their initial surgery at other centres. Forty-one percent $(n=48)$ were female and the median age at diagnosis was 60 years old (range 18-86 years). Two patients had more than one biopsy undertaken due to non-diagnostic biopsy results.

This article is protected by copyright. All rights reserved. 
Based on final surgical pathology, the diagnosis was WDLPS $(22 \%, n=26)$, WD/DDLPS (37\%, n=43), LMS (14\%, n=16), myxoid LPS (2\%, n=2), UPS (4\%, n=5), SFT $(9 \%, n=10)$, other sarcoma $(6 \%, n=7)$ or a benign process $(5 \%, n=6)$ (see table 3). Comparison between percutaneous biopsy and final histopathology from surgical resection was analysed based on histopathological subtype and sensitivity, specificity, positive and negative predictive values were calculated for each (see table 4). Biopsy accuracy varied with histopathological subtype, however, overall agreement between biopsy and final pathology was seen in $61 \%$ of cases $(\kappa=0.57$, $95 \%$ Cl 0.45 to 0.69$)$.

Sensitivity was highest for myxoid liposarcoma $(100 \%, 95 \% \mathrm{Cl} 0.16$ to 1.00$)$ followed by WDLPS (85\%, 95\% Cl 0.65 to 0.96$)$ and LMS (81\%, 95\% Cl 0.54 to 0.96$)$.

Despite the high sensitivity, PPV for WDLPS was only $58 \%$ as 13 tumours that were initially biopsied as WDLPS were found to have a de-differentiated component on final histopathology. Sensitivity was lowest for detecting DDLPS (40\%, 95\% CI 0.25 to 0.56 ) and in $56 \%$ of cases where the initial biopsy was discordant with final histopathology, the final diagnosis was DDLPS $(n=26)$. Despite the low sensitivity, the specificity of percutaneous biopsy for DDLPS was high $(92 \%, 95 \% \mathrm{Cl} 0.83$ to 0.97) demonstrating that when de-differentiation was identified on percutaneous biopsy, that this was likely to be a true finding. 
Of the 26 cases of DDLPS that were not correctly diagnosed on percutaneous biopsy, half of these were initially thought to be WDLPS and the rest were either classified as UPS (23\%), SFT (4\%), indeterminate/other sarcoma (11.5\%) or benign tumours (11.5\%) on percutaneous biopsy. When considering liposarcoma as a single group therefore (WDLPS and WD/DDLPS together), sensitivity of percutaneous improved to $81 \%$ (95\% Cl 0.70 to 0.90$)$ and specificity was $90 \%$ (95\% Cl 0.77 to 0.97).

\section{Subgroup analysis}

Subgroup analysis was undertaken according two main factors which may have impacted upon the concordance between percutaneous biopsy and final histopathology (see table 5). The first factor was use of PET/CT scan prior to biopsy and the second was use of fluorescence in-situ hybridisation testing on core biopsies.

\section{PET/CT scan prior to biopsy}

In this study, ${ }^{18} \mathrm{~F}$-fluorodeoxyglucose positron emission tomography / computed tomography (FDG-PET/CT) imaging was undertaken prior to percutaneous biopsy in $43 \%$ of cases $(n=50)$. The agreement between biopsy and final pathology in those who underwent PET/CT scan prior to biopsy was substantial $(\kappa=0.69,95 \% \mathrm{Cl} 0.53$ to 0.84) whilst for those who did not undergo PET/CT scan was moderate $(\kappa=0.48$, $95 \% \mathrm{Cl} 0.30$ to 0.65$)$. The sensitivity of biopsy for diagnosing WDLPS, DDLPS and 
LPS combined was higher for all groups than when PET/CT was undertaken prior to biopsy however, despite biopsy sensitivity increasing, PPV for WDLPS remained low $59 \%$ ( $\mathrm{Cl} 0.36$ to 0.79 ) suggesting that PET/CT did not improve the detection of dedifferentiated components in WD/DDLPS.

\section{Fluorescence In-Situ Hybridisation (FISH)}

FISH testing for the MDM2 (murine double minute) gene was undertaken on percutaneous biopsies in 78 cases (67\%). There was substantial agreement between biopsy and final pathology in those who had FISH testing undertaken on their biopsy ( $\kappa=0.77, \mathrm{Cl} 0.65$ to 0.90 ) whilst fair agreement for those who did not have FISH testing undertaken ( $\kappa=0.25, \mathrm{Cl} 0.06$ to 0.44 ). The main benefit of FISH testing is in identifying DDLPS from UPS with similar undifferentiated appearance histologically, but the presence of MDM2 amplification is diagnostic of DDLPS. It was noted that in 6 cases, patients were initially diagnosed with UPS on percutaneous biopsy and subsequently diagnosed with WD/DDLPS on final histopathology from surgical resection. None of these 6 cases had FISH testing undertaken on their percutaneous biopsies.

\section{FISH and PET/CT combined}

There were 33 cases where both PET/CT scan prior to biopsy and FISH were undertaken. These 33 cases demonstrated almost perfect agreement between biopsy and final pathology ( $\kappa=0.85,95 \% \mathrm{Cl} 0.70$ to 1.00$)$. Of these 33 cases, the 
sensitivity of biopsy for WDLPS was $100 \%$ (95\% Cl 0.66 to 1.00), for DDLPS the sensitivity was $60 \%$ ( $95 \% \mathrm{Cl} 0.32$ to 0.84$)$ and for LPS as a combined entity the sensitivity was $100 \%$ (95\% Cl 0.86 to 1.00$)$. Even when both PET/CT and FISH were undertaken, there were still 7 cases where DDLPS was initially diagnosed as WDLPS and the PPV of WDLPS was unchanged (56\%, Cl 0.30 to 0.80). In the 6 cases in which DDLPS was initially diagnosed as UPS, no patients had both PET/CT and FISH undertaken.

\section{Discussion}

Retroperitoneal sarcoma is a rare and heterogenous diagnosis for which the mainstay of treatment remains surgical resection. Tumour behaviour varies depending on pathological subtype, and particularly, on histological grade and thus determining correct diagnosis is important for optimal prognostication and management. There are some studies assessing the accuracy of biopsy in diagnosing retroperitoneal sarcoma, but the published evidence still remains limited. The findings of this study however are in keeping with those already published in the broader literature. A recent paper by Almond et $\mathrm{al}^{7}$ looking at diagnostic accuracy of percutaneous biopsy in retroperitoneal sarcoma at two major sarcoma centres in Europe reported an overall concordance rate of $67 \%(\kappa=0.60)$ for biopsy and final histopathology for retroperitoneal sarcomas. The subgroup analysis reported by Almond reported findings similar to this study with a sensitivity of $95 \%$ for WDLPS, 
$40 \%$ for DDLPS and $85 \%$ for LPS combined. Ikoma et $\mathrm{al}^{8}$ similarly concluded that percutaneous biopsy had poor accuracy for correctly diagnosing DDLPS (36.5\% vs $85.1 \%$ accuracy of diagnosing WDLPS).

There are several reasons as to why diagnosis of DDLPS is likely to be less accurate on percutaneous biopsy than other subtypes of sarcoma. DDLPS by nature is a heterogenous tumour type, characterised by the presence of de-differentiated tumour elements within a WDLPS. As such, sampling error may occur and lead to biopsies identifying only well-differentiated components, thus failing to diagnose DDLPS (see figure 1). Additionally, the de-differentiated tumour components can have variable morphology also and thus may be mistaken for other tissue diagnoses. Increasingly, adjunct diagnostic tools are being used to assist with increasing biopsy accuracy. Two particular diagnostic adjuncts at present are FDG-PET/CT scan and FISH testing for MDM2 gene amplification.

At PMCC, PET/CT scan is undertaken as a routine part of work up for patients with sarcoma (although as seen in this study, timing of PET/CT relative to biopsy can be inconsistent based on the scheduling availability with only $43 \%$ being undertaken prior to biopsy). FDG-PET/CT imaging provides high grade imaging of sarcomas and can be useful for providing an overall picture of heterogeneity in sarcoma tissues. Cells with a high metabolic activity (e.g. malignant or inflammatory cells) take up FDG to a greater extent than less active cells. Several studies report a positive 
correlation between tumour grade and Standardised Uptake Value Maximum (SUVmax) suggesting that FDG-PET/CT may be useful for distinguishing between high and low grade sarcomas but it may not provide useful additional information for discriminating between benign pathologies and low grade soft tissue sarcomas ${ }^{9-14}$. The literature suggests that FDG-PET/CT SUVmax is a prognostic factor with increased SUVmax being associated with poorer overall survival and disease progression in sarcoma ${ }^{15}$. In theory, PET/CT scan may assist in improving accuracy of percutaneous biopsy by allowing clinicians to see and target the most metabolically active components of the tumour ${ }^{15}$. This study observed a small increase in the sensitivity of percutaneous biopsy across all sarcoma histologic subtypes when PET/CT scan was undertaken prior to biopsy. Despite the increase in sensitivity however, PET/CT did not assist with differentiating between WDLPS and DDLPS in this cohort. Notwithstanding the limitations of FDG-PET, this study suggests that PET scan may provide additional benefit in improving diagnostic accuracy and prognostic stratification in patients with RPS and therefore it should be considered in the management of patients with RPS and be scheduled prior to tumour biopsy.

Fluorescence in situ hybridisation (FISH) has increasingly been used to assist in diagnosis of RPS over the last 10 years. FISH is a cytogenetic test which utilises a fluorescent probe to identify the presence of specific gene sequences ${ }^{16}$. Amplification of the MDM2 gene is characteristic of WDLPS and WD/DDLPS and 
can assist in differentiating these pathologies from other sarcomatous subtypes (e.g. pleomorphic sarcoma) or benign tumours with a similar morphological appearance such as renal angiomyolipoma ${ }^{17-20}$. As observed in this study, the sensitivity of biopsy for detecting LPS as a whole increased to 94\% when FISH was undertaken on percutaneous biopsies. Interestingly however, in this study, a large number of the tumours that were incorrectly diagnosed on percutaneous biopsy were DDLPS that were initially diagnosed as WDLPS and thus FISH was not a useful test for distinguishing between these pathologies.

Myxoid liposarcoma is another sarcoma subtype in which molecular testing for genetic mutations can assist accurate diagnosis. Myxoid liposarcomas typically demonstrate abnormalities in the FUS-DDIT3 (fused in sarcoma) gene, and presence of such abnormalities can help to differentiate between myxoid liposarcoma and other tumours with a similar histological appearance ${ }^{21}$. In this study there were only four patients with a pre-operative diagnosis of myxoid liposarcoma. In two of these cases, the diagnosis was revised on final pathology (one received a final diagnosis of myoepithelial carcinoma and the other a diagnosis of myxofibrosarcoma). Neither of these cases underwent molecular testing on the initial biopsy specimen and occurred in the early period of the study cohort. These findings suggest that a pre-operative diagnosis of myxoid liposarcoma requires confirmatory molecular pathology (as is now routine at our institution). 
An important component of the optimal multidisciplinary management of patients with a new diagnosis of suspected sarcoma is pathology review by an expert sarcoma pathologist. The NICE guidelines for management of sarcoma recommend that all patients with a suspected or confirmed diagnosis of sarcoma should have their care discussed at a specialist sarcoma multidisciplinary meeting ${ }^{22,23}$. As part of this process, it is recommended that pre-operative biopsy is undertaken by a specialist with an interest in sarcoma management and that histopathology is reviewed by an expert pathologist. All patients with a suspected diagnosis of retroperitoneal sarcoma should be referred to a sarcoma specialist centre for review of imaging and confirmation of diagnosis via tissue biopsy. These patients should be reviewed at a sarcoma multidisciplinary meeting to obtain expert, consensus opinion about their management.

All patients with a suspected retroperitoneal sarcoma should have preoperative core needle biopsy to clarify the diagnosis and allow optimal therapy. The biopsy is important to rule out other (non-sarcoma) pathologies as well as to identify the histological subtype and grade of sarcoma. A limitation of this study is that the sampled population only included patients with a definitive diagnosis of RPS. As such, this study did not include patients with suspected RPS on imaging where the preoperative biopsy was benign or consistent with an alternative pathology. Future studies that include this population will provide a clear understanding of the utility of core biopsy in patients with suspected RPS. 


\section{Conclusion}

Retroperitoneal sarcomas are a rare and heterogenous group of soft tissue tumours. Management of these tumours relies heavily on percutaneous core biopsy to guide treatment plans, however, the accuracy of biopsy results varies between histopathological subtypes of retroperitoneal sarcoma. A significant number of patients with a well differentiated liposarcoma will have a dedifferentiated component identified at the time of resection that was not identified on initial biopsy. While adjunct investigations such as pre-biopsy PET/CT scan and use of FISH testing on biopsies may improve biopsy sensitivity for subtypes of sarcoma, neither one improves the detection of dedifferentiated components on biopsy.

\section{Acknowledgements}

The authors are grateful for the support of Jasmine Mar and the Australia New Zealand Sarcoma Association for their support of the retroperitoneal sarcoma registry and to Mr Michael A Henderson for his contribution to the retroperitoneal sarcoma registry and manuscript editing.

\section{Disclosure statement}

The authors have no conflict of interest to disclose. 
This article is protected by copyright. All rights reserved. 


\section{References}

[1] Dumitra S, Gronchi A. The Diagnosis and Management of Retroperitoneal Sarcoma. Oncology 2018; 32:464-9.

[2] Trans-Atlantic RPS Working Group. Management of Primary Retroperitoneal Sarcoma (RPS) in the Adult: A Consensus Approach From the Trans-Atlantic RPS Working Group. Annals Of Surgical Oncology 2015; 22:256-63.

[3] Mota M, Bezerra R, Garcia M. Practical approach to primary retroperitoneal masses in adults. Radiol Bras 2018; 51:391-400.

[4] Cohen J. Weighted kappa: Nominal scale agreement provision for scaled disagreement or partial credit. Psychological Bulletin 1968; 70:213-20.

[5] Fletcher C, Bridge, J, Hogendoorn P, Mertens F. WHO Classification of Tumours of Soft Tissue and Bone. Fourth Edition. Lyon, France: IARC Press, 2013.

[6] Snow H, Hitchen $\mathrm{T}$, Head J et al. Treatment of patients with primary retroperitoneal sarcoma: predictors of outcome from an Australian specialist sarcoma centre. ANZ Journal of Surgery 2018; 88:1151-7.

[7] Almond L, Tirotta F, Tattersall $\mathrm{H}$ et al. Diagnostic accuracy of percutaneous biopsy in retroperitoneal sarcoma. British Journal of Surgery 2019:395.

[8] Ikoma N, Torres K, Somaiah N et al. Accuracy of Preoperative Percutaneous Biopsy for the Diagnosis of Retroperitoneal Liposarcoma Subtypes. United States: Springer Science + Business Media, 2015; 1068.

[9] Alford S, Choong P, Chander S et al. Value of PET scan in patients with retroperitoneal sarcoma treated with preoperative radiotherapy. European Journal of Surgical Oncology 2012; 38:176-80.

[10] Brenner W, Eary J, Hwang W et al. Risk assessment in liposarcoma patients based on FDG PET imaging. European Journal of Nuclear Medicine and Molecular Imaging 2006; 33:1290-5.

[11] Nieweg O, van Ginkel R, Hoekstra H, Paans A, Molenaar W et al. . Fluorine-18fluorodeoxyglucose PET imaging of soft-tissue sarcoma. J Nucl Med 1996; 37:257-61.

[12] Schwarzbach M, Dimitrakopoulou-Strauss A, Willeke F et al. Clinical value of [18-F]] fluorodeoxyglucose positron emission tomography imaging in soft tissue sarcomas. Ann Surg 2000; 231:380-6.

[13] Tateishi U, Yamaguchi, U, Seki, K et al. Glut-1 expression and enhanced glucose metabolism are associated with tumour grade in bone and soft tissue sarcomas: a prospective evaluation by [18F]fluorodeoxyglucose positron emission tomography. Eur J Nucl Med Mol Imaging 2006; 33:683.

[14] Ioannidis J, Lau J. 18F-FDG PET for the Diagnosis and Grading of Soft-Tissue Sarcoma: A Meta-Analysis. Journal of nuclear medicine : official publication, Society of Nuclear Medicine 2003; 44:717-24.

[15] Eary J, Conrad E. PET imaging: update on sarcomas. Oncology 2007; 21:249-52. 
[16] Asif A, Mushtaq S, Hassan U, et al. Fluorescence in Situ Hybridization (FISH) for Differential Diagnosis of Soft Tissue Sarcomas. Asian Pac J Cancer Prev 2018; 19:655-60. [17] Clay MR, Martinez AP, Weiss SW, Edgar MA. MDM2 Amplification in Problematic Lipomatous Tumors: Analysis of FISH Testing Criteria. The American Journal of Surgical Pathology 2015; 39:1433-9.

[18] Kimura H, Yamamoto N, Shirai T et al. Detection of MDM2 gene amplification in soft tissue sarcoma by FISH. Journal of Clinical Oncology 2014; 32:10562-.

[19] Thway K, Wang J, Swansbury J, Min T, Fisher C. Fluorescence In Situ Hybridization for MDM2 Amplification as a Routine Ancillary Diagnostic Tool for Suspected Well-

Differentiated and Dedifferentiated Liposarcomas: Experience at a Tertiary Center. Sarcoma 2015; 2015.

[20] Weaver J, Downs-Kelly E, Goldblum J et al. Fluorescence in situ hybridization for MDM2 gene amplification as a diagnostic tool in lipomatous neoplasms. Modern Pathology 2008; 21:943.

[21] Yu J, Colborne S, Hughes C, Morin G, Nielsen T. The FUS-DDIT3 Interactome in Myxoid Liposarcoma. Neoplasia 2019; 21:740-51.

[22] National Institute for Health and Clinical Excellence (NICE). Improving outcomes for people with sarcoma. The Manual. NICE 2006.

[23] Cancer Council Australia Sarcoma Guidelines Working Party. Clinical practice guidelines for the management of adult onset sarcoma. Sydney: Cancer Council Australia. Edition., cited 28 December 2019].Available from:

https://wiki.cancer.org.au/australiawiki/index.php?oldid=138276 


\section{Figure Legends}

Figure 1: Case example of a retroperitoneal sarcoma where initial biopsy suggested a diagnosis of WDLPS with surgical resection specimen demonstrating $20 \%$ dedifferentiation making the final diagnosis WD/DDLPS
a) Pre-biopsy CT scan
b) Pre-biopsy PET scan
c) Surgical specimen 
Table 1: Agreement between biopsy and final histopathology (Cohen's kappa)

\begin{tabular}{|l|l|l|}
\hline Subgroup & Kappa score $(95 \% \mathrm{Cl})$ & Agreement \\
\hline All & $0.57(0.45$ to 0.69$)$ & Moderate agreement \\
\hline All subgroups with PET pre-biopsy & $0.69(0.53$ to 0.84$)$ & Substantial agreement \\
\hline All subgroups without PET pre-biopsy & $0.48(0.30$ to 0.65$)$ & Moderate agreement \\
\hline All subgroups with FISH on biopsy & $0.77(0.65$ to 0.90$)$ & Substantial agreement \\
\hline All subgroups without FISH on biopsy & $0.25(0.06$ to 0.44$)$ & Fair agreement \\
\hline All subgroups with both PET and FISH & $0.85(0.70$ to 1.00$)$ & Almost perfect agreement \\
\hline
\end{tabular}


Table 2: Demographics

\begin{tabular}{|l|l|}
\hline Characteristic & Number \\
\hline Sex & \\
Male & $69(58.97 \%)$ \\
Female & $48(41.03 \%)$ \\
\hline Median age & 60 years (range 18-86mm) \\
\hline Median tumour size & \\
\hline PET pre-biopsy & $142 \mathrm{~mm}($ range $23-380 \mathrm{~mm})$ \\
Yes & \\
No & $49(41.88 \%)$ \\
\hline FISH on biopsy & $68(58.12 \%)$ \\
Yes & \\
No & $78(66.67 \%)$ \\
Unknown & $26(22.22 \%)$ \\
\hline Neoadjuvant radiotherapy & $13(11.11 \%)$ \\
\hline
\end{tabular}

This article is protected by copyright. All rights reserved. 
This article is protected by copyright. All rights reserved. 
Table 3: Percutaneous biopsy and final histopathology

\begin{tabular}{|c|c|c|c|c|c|c|c|c|c|c|c|}
\hline \multirow[b]{2}{*}{ Biopsy result } & \multicolumn{11}{|c|}{ Histopathology from surgical resection n (sensitivity \%) } \\
\hline & WDLPS & DDLPS & LMS & $\begin{array}{l}\text { Myxoid } \\
\text { LPS }\end{array}$ & UPS & SFT & $\begin{array}{l}\text { Other } \\
\text { sarcoma }\end{array}$ & $\begin{array}{l}\text { non- } \\
\text { sarcoma } \\
\text { malignancy }\end{array}$ & $\begin{array}{l}\text { benign } \\
\text { tumour }\end{array}$ & $\begin{array}{l}\text { non- } \\
\text { diagnostic }\end{array}$ & Total \\
\hline WDLPS & $22(85 \%)$ & 13 & 0 & 0 & 0 & 1 & 0 & 0 & 2 & 0 & 38 \\
\hline DDLPS & 4 & \begin{tabular}{|l|}
17 \\
$(40 \%)$ \\
\end{tabular} & 1 & 0 & 0 & 1 & 0 & 0 & 0 & 0 & 23 \\
\hline LMS & 0 & 0 & \begin{tabular}{|l|}
13 \\
$(81 \%)$ \\
\end{tabular} & 0 & 0 & 0 & 0 & 0 & 0 & 0 & 13 \\
\hline Myxoid LPS & 0 & 0 & 0 & \begin{tabular}{|l}
2 \\
$(100 \%)$ \\
\end{tabular} & 0 & 0 & 1 & 1 & 0 & 0 & 4 \\
\hline UPS & 0 & 6 & 1 & 0 & $\begin{array}{l}2 \\
(40 \% \\
) \\
\end{array}$ & 0 & 0 & 0 & 0 & 0 & 9 \\
\hline SFT & 0 & 1 & 0 & 0 & 0 & $\begin{array}{l}7 \\
(70 \\
\%) \\
\end{array}$ & 0 & 0 & 0 & 0 & 8 \\
\hline Other sarcoma & 0 & 3 & 0 & 0 & 1 & 0 & $4(57 \%)$ & 0 & 0 & 1 & 9 \\
\hline $\begin{array}{l}\text { non-sarcoma } \\
\text { malignancy }\end{array}$ & 0 & 0 & 0 & 0 & 1 & 0 & 1 & 0 & 0 & 0 & 2 \\
\hline
\end{tabular}




\begin{tabular}{|l|l|l|l|l|l|l|l|l|l|l|l|}
\hline benign tumour & 0 & 3 & 0 & 0 & 0 & 1 & 0 & 0 & 4 & $\begin{array}{l}4 \\
(67 \%)\end{array}$ & 0 \\
\hline non-diagnostic & 0 & 0 & 1 & 0 & 1 & 0 & 1 & 0 & 0 & 0 \\
\hline Total & 26 & 43 & 16 & 2 & 5 & 10 & 7 & 1 & 6 & 1 \\
\hline
\end{tabular}


Table 4: Sensitivity, specificity, PPV and NPV by histological subtype

\begin{tabular}{|l|l|l|l|l|}
\hline $\begin{array}{l}\text { Histology on biopsy } \\
\text { ( } \mathrm{n}=\text { total on biopsy) }\end{array}$ & Sensitivity $(95 \% \mathrm{Cl})$ & Specificity $(95 \% \mathrm{Cl})$ & $\mathrm{PPV}(95 \% \mathrm{Cl})$ & $\mathrm{NPV}(95 \% \mathrm{Cl})$ \\
\hline $\begin{array}{l}\text { Liposarcoma combined } \\
(\mathrm{n}=61)\end{array}$ & $0.81(0.70$ to 0.90$)$ & $0.90(0.77$ to 0.97$)$ & $0.92(0.82$ to 0.97$)$ & $0.90(0.77$ to 0.97$)$ \\
\hline WDLPS $(\mathrm{n}=38)$ & $0.85(0.65$ to 0.96$)$ & $0.82(0.73$ to 0.90$)$ & $0.58(0.41$ to 0.74$)$ & $0.95(0.88$ to 0.99$)$ \\
\hline DDLPS $(\mathrm{n}=23)$ & $0.40(0.25$ to 0.56$)$ & $0.92(0.83$ to 0.97$)$ & $0.74(0.52$ to 0.90$)$ & $0.72(0.61$ to 0.81$)$ \\
\hline LMS $(\mathrm{n}=13)$ & $0.81(0.54$ to 0.96$)$ & $1.00(0.97$ to 1.00$)$ & $1.00(0.75$ to 1.00$)$ & $0.97(0.93$ to 0.99$)$ \\
\hline Myxoid LPS $(n=4)$ & $1.00(0.16$ to 1.00$)$ & $0.98(0.94$ to 1.00$)$ & $0.50(0.07$ to 0.93$)$ & $1.00(0.97$ to 1.00$)$ \\
\hline UPS $(n=9)$ & $0.40(0.05$ to 0.85$)$ & $0.94(0.88$ to 0.97$)$ & $0.22(0.03$ to 0.60$)$ & $0.97(0.92$ to 0.99$)$ \\
\hline SFT $(n=8)$ & $0.70(0.35$ to 0.93$)$ & $0.99(0.95$ to 1.00$)$ & $0.88(0.47$ to 1.00$)$ & $0.97(0.92$ to 0.99$)$ \\
\hline Other sarcoma $(n=9)$ & $0.57(0.18$ to 0.90$)$ & $0.95(0.90$ to 0.99$)$ & $0.44(0.14$ to 0.79$)$ & $0.97(0.92$ to 0.99$)$ \\
\hline Benign tumour $(n=8)$ & $0.67(0.22$ to 0.96$)$ & $0.96(0.91$ to 0.99$)$ & $0.50(0.16$ to 0.84$)$ & $0.98(0.94$ to 1.00$)$ \\
\hline
\end{tabular}


Table 5: Sensitivity, specificity, PPV and NPV according to adjunct pre-biopsy diagnostic testing

\begin{tabular}{|l|l|l|l|l|}
\hline $\begin{array}{l}\text { Diagnosis on final } \\
\text { histopathology and } \\
\text { adjunct test }(\mathrm{n}=\text { total } \\
\text { biopsy) }\end{array}$ & Sensitivity $(95 \% \mathrm{Cl})$ & Specificity $(95 \% \mathrm{Cl})$ & PPV (95\% Cl) & NPV (95\% Cl) \\
\hline WDLPS $(n=38)$ & $0.85(0.65$ to 0.96$)$ & $0.82(0.73$ to 0.90$)$ & $0.58(0.41$ to 0.74$)$ & $0.95(0.88$ to 0.99$)$ \\
PET $(n=22)$ & $1.00(0.75$ to 1.00$)$ & $0.76(0.59$ to 0.88$)$ & $0.59(0.36$ to 0.79$)$ & $1.00(0.88$ to 1.00$)$ \\
FISH $(n=27)$ & $0.80(0.56$ to 0.94$)$ & $0.81(0.69$ to 0.90$)$ & $0.59(0.39$ to 0.78$)$ & $0.92(0.81$ to 0.98$)$ \\
PET + FISH $(n=16)$ & $1.00(0.66$ to 1.00$)$ & $0.71(0.49$ to 0.87$)$ & $0.56(0.30$ to 0.80$)$ & $1.00(0.80$ to 1.00$)$ \\
\hline DDLPS $(n=23)$ & $0.40(0.25$ to 0.56$)$ & $0.92(0.83$ to 0.97$)$ & $0.74(0.52$ to 0.90$)$ & $0.72(0.61$ to 0.81$)$ \\
PET $(n=11)$ & $0.50(0.28$ to 0.72$)$ & $1.00(0.88$ to 1.00$)$ & $1.00(0.72$ to 1.00$)$ & $0.72(0.55$ to 0.85$)$ \\
FISH $(n=20)$ & $0.56(0.35$ to 0.76$)$ & $0.89(0.77$ to 0.96$)$ & $0.70(0.46$ to 0.88$)$ & $0.81(0.69$ to 0.90$)$ \\
PET + FISH $(n=9)$ & $0.60(0.32$ to 0.84$)$ & $1.00(0.81$ to 1.00$)$ & $1.00(0.66$ to 1.00$)$ & $0.75(0.53$ to 0.90$)$ \\
\hline LPS $(n=61)$ & $0.81(0.70$ to 0.90$)$ & $0.90(0.77$ to 0.97$)$ & $0.92(0.82$ to 0.97$)$ & $0.90(0.77$ to 0.97$)$ \\
PET $(n=33)$ & $0.91(0.77$ to 0.98$)$ & $0.93(0.68$ to 1.00$)$ & $0.97(0.84$ to 1.00$)$ & $0.82(0.57$ to 0.96$)$ \\
FISH $(n=47)$ & $0.94(0.82$ to 0.99$)$ & $0.90(0.74$ to 0.98$)$ & $0.94(0.82$ to 0.99$)$ & $0.90(0.74$ to 0.98$)$ \\
PET + FISH $(n=25)$ & $1.00(0.86$ to 1.00$)$ & $0.89(0.52$ to 1.00$)$ & $0.96(0.80$ to 1.00$)$ & $1.00(0.63$ to 1.00$)$ \\
\hline
\end{tabular}

This article is protected by copyright. All rights reserved. 


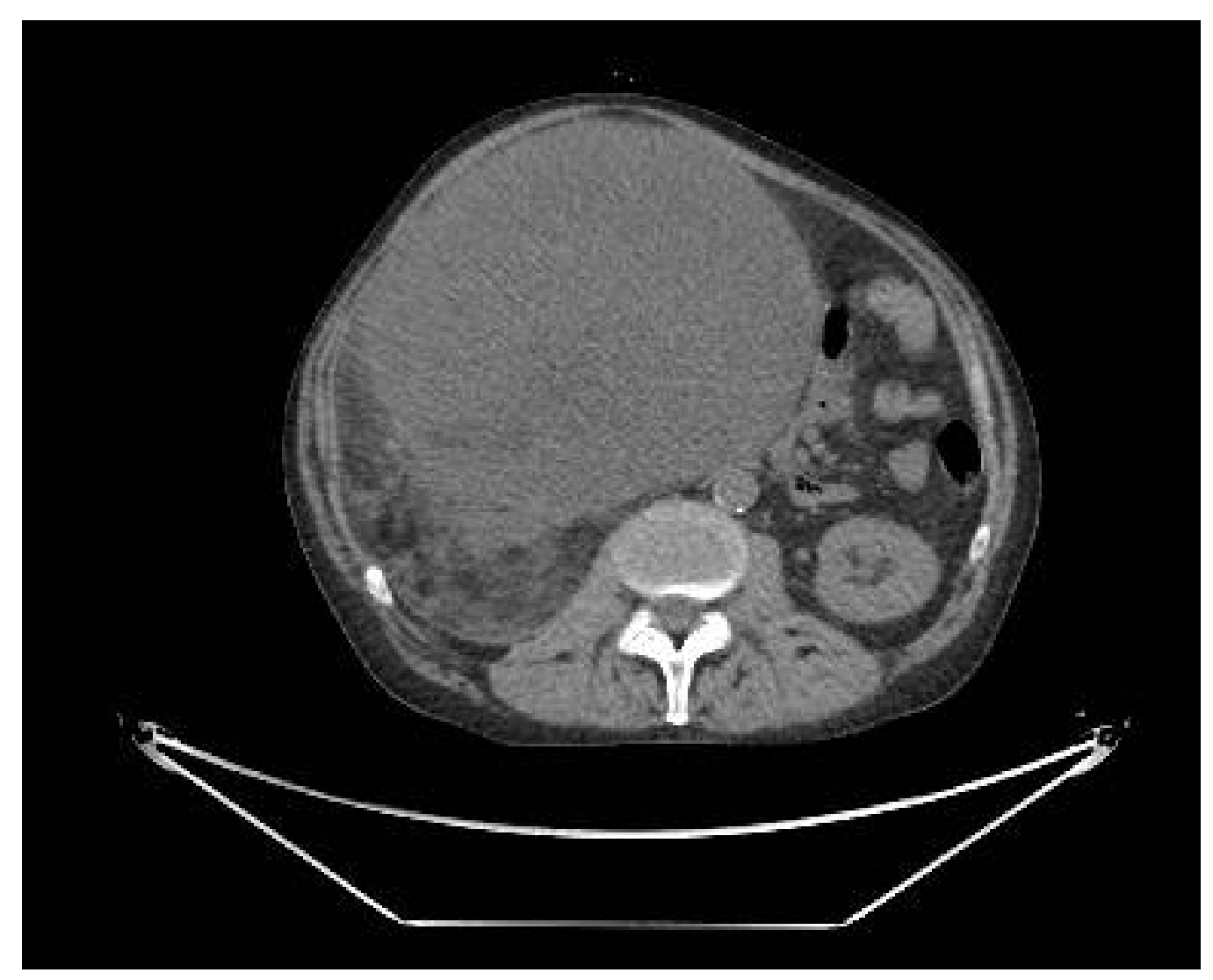

ANS_15723_YoungFig1a.tiff

This article is protected by copyright. All rights reserved. 


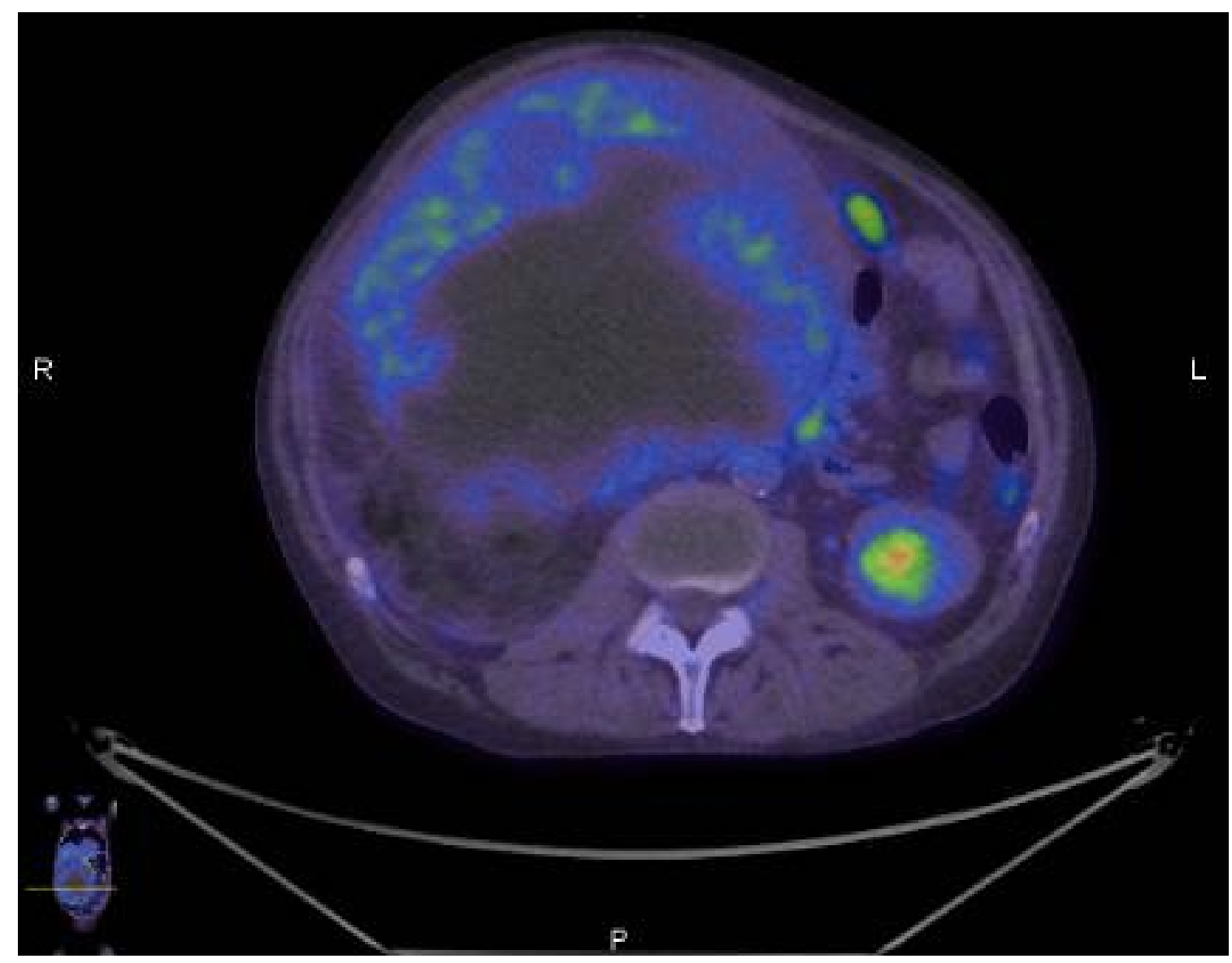

ANS_15723_YoungFig1b.tiff

This article is protected by copyright. All rights reserved. 


\section{University Library}

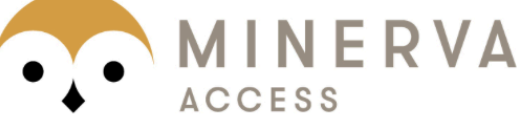

A gateway to Melbourne's research publications

Minerva Access is the Institutional Repository of The University of Melbourne

Author/s:

Young, R;Snow, H;Hendry, S;Mitchell, C;Slavin, J;Schlicht, S;Na, L;Hofman, MS;Gyorki, DE

Title:

Correlation between percutaneous biopsy and final histopathology for retroperitoneal sarcoma: a single-centre study

Date:

2020-02-16

Citation:

Young, R., Snow, H., Hendry, S., Mitchell, C., Slavin, J., Schlicht, S., Na, L., Hofman, M. S. \& Gyorki, D. E. (2020). Correlation between percutaneous biopsy and final histopathology for retroperitoneal sarcoma: a single-centre study. ANZ JOURNAL OF SURGERY, 90 (4), pp.497-502. https://doi.org/10.1111/ans.15723.

Persistent Link:

http://hdl.handle.net/11343/275420 